\title{
RESEARCH
}

\section{Exploring Hidden Messages About Pharmacist Roles In Student-Designed Orientation T- Shirts}

\author{
Mike Chong, MEd, Beverly FitzPatrick, PhD \\ Memorial University, St. John's, Newfoundland and Labrador, Canada \\ Corresponding Author: Mike Chong, Memorial University, School of Pharmacy, 300 Prince Philip Dr., St. John's, NL, A1B 3 V6. \\ Tel: 709-864-4736. Email: mchong@mun.ca
}

Submitted July 15, 2021; accepted October 22, 2021; ePublished November 2021

Objective. To develop an understanding of how images and symbols on student-designed orientation t-shirts represent students' beliefs about pharmacists' roles in practice.

Methods. An exploratory qualitative study underpinned by perspectives on hidden curriculum and discursive practices was conducted at one Canadian pharmacy school. First year students wrote responses to prompts about the t-shirts at the start and end of the school year. Concurrently, semi-structured interviews and focus groups were held with faculty, staff, and $2^{\text {nd }}-4^{\text {th }}$ year students. Six t-shirts from 2014-2019 were used for discussion. Data were independently coded by the two authors and themes were developed.

Results. Students and faculty had mostly similar thoughts about the practice of giving orientation t-shirts and the messages on them. Many pointed to the drug-related aspects of pharmacists' roles and did not question this representation until explicitly prompted. Relatedly, most participants did not suggest that the t-shirts should emphasize the pharmacistpatient relationship and care provision. And while there were mixed thoughts about the business logo on the t-shirts, participants were often unperturbed by sponsorship or its potential effects on students.

Conclusion. This study showed that despite national educational outcomes advocating the care provider role, these representations of pharmacy and pharmacists' roles still focus on drug expertise and drug provision. By revealing thoughts about the t-shirt images, the study highlighted how, despite explicit teaching of pharmacists' roles and the expanding scope of pharmacy, discursive practices in pharmacy education exist and form part of the hidden curriculum.

Keywords: symbolism, discursive practices, hidden curriculum, pharmacist roles

\section{INTRODUCTION}

Pharmacy education should have a degree of alignment with expectations of pharmacy practice. Over the past two decades, an expanded scope of practice in Canada focused on patient-centered care has occurred nationwide.

Complementing these national initiatives, the conceptual framework for Canadian entry-to-practice educational outcomes emphasizes that the role of care provider is the core of pharmacy, similar to the United States. ${ }^{1,2}$ Thus, there is alignment between pharmacy practice and educational outcomes for schools in Canada and the US.

However, students receive implicit messages that may be contrary to and undermine the intents of the explicit curriculum. ${ }^{3-5}$ In this study we attempted to understand some of these implicit messages emanating from what Foucault terms discursive practices - socio-cultural practices that occur on a daily or regular basis and work together to create truth in our minds. ${ }^{6,7}$ These practices play an important role in identity formation, comprising the words and actions that form our instruction, and the nuances that are often associated with our word choice and actions, especially in the minds of students. ${ }^{6}$ We wanted to know what implicit messages our students were receiving, whether they matched formal curriculum goals, and what truths were being formed about pharmacists' roles. This is particularly important because pharmacy education may be contributing inadvertently to the complex and perhaps conflicting pharmacists' roles and identities. ${ }^{8}$ Elvey et al. identified 9 pharmacist identities, suggesting that pharmacists are uncertain about their identities or have a diversity of roles. ${ }^{9}$ Furthermore, debates exist about the role pharmacists play in healthcare, both within and between academics, practicing pharmacists, and students. ${ }^{10,11}$ This ambiguity of professional identity has been proposed as a significant barrier to internalizing and articulating pharmacists' roles and scope of practice. ${ }^{12,13}$

To explore how discursive practices might contribute to this issue of pharmacist identity, we analyzed messages students were getting from first year orientation $t$-shirts that are intended to represent pharmacy. These t-shirts usually incorporate a pop culture design, pharmacy business logos, pharmacy related symbols, and drug products such as pills or capsules. Symbols in pharmacy have a historical and traditional significance passed on over generations of pharmacists. Some of them, such as the Bowl of Hygeia, have been understood as symbols of health but also indicate the presence of a 
pharmacy. ${ }^{14}$ Others like the mortar and pestle represent the tools of the apothecary or the compounding of medications. ${ }^{15}$ Similarly, the R symbol may have started as a Latin directive for "Recipe: Take thou!" but has now become synonymous with prescription. ${ }^{14,16}$ Bailey explains that symbols are ideographic in that they can be understood quickly without words and represent deeper meanings and contexts. ${ }^{16} \mathrm{We}$ thought that because students choose the t-shirt designs, we could gain insight into students' beliefs about pharmacists' roles, and that faculty and staff interpretations would further enhance our understandings. There is limited research on how implicit messages in pharmacy education affect students' beliefs and role formations. ${ }^{17}$ This study was intended to illuminate how orientation t-shirts, as a representation of hidden messages and symbolism, can portray students' beliefs and individual truths about the role of pharmacists.

Objective. The objective of this study was to develop an understanding of how images and symbols on student-designed orientation t-shirts represent students' beliefs about pharmacists' roles in practice.

\section{METHODS}

We used a qualitative methodology to explore how images on orientation t-shirts for first year pharmacy students in one Canadian school align with nationwide goals of pharmacy education in terms of providing patient care, including the role of business sponsorship. ${ }^{18}$ Our methodology was informed by Foucault's notion of discursive practices because representation of pharmacists on the t-shirts and the experiences that students and faculty embody when seeing or donning these t-shirts reflect beliefs and identities about pharmacy. ${ }^{6}$ Some of these beliefs may be implicit or in the subconscious minds of students and faculty, such that they may not explicitly think about the messages of the t-shirts related to pharmacists' roles. But, using a qualitative approach allowed us to inquire deeply into participants' thoughts and feelings about the meanings ascribed to the images and provoke the participants to probe into their subconscious thoughts that may result from discursive practices in pharmacy education.

We used purposive, criterion sampling to select groups of people that fit our study's purpose. ${ }^{18}$ Within these groups, we used maximum variation sampling to recruit faculty, staff, orientation committee members, and students from all years of the pharmacy program so we could obtain information from as diverse a sample as possible that met the criterion. ${ }^{18}$

First year students in 7/10 Canadian pharmacy schools receive orientation t-shirts. The school in our study has an orientation committee consisting of two senior students and two shadowing junior students, who design the t-shirts and find sponsorship. The senior students look for designs, usually on the internet, that they deem appropriate for new students. These two students choose a final design, sometimes with input from the juniors. Approval is sought from the school and then a local printshop produces the t-shirts. We used six t-shirts from 2014-2019 as a focal point for discussion. The shirts had various images and text referencing superheroes, drugs, pharmacists, or the pharmacy degree. Of note, the sponsor's logo was on all the t-shirts and usually was prominent on the back of the shirt. For confidentiality, we have not indicated the sponsor's name. Since we used the physical t-shirts or colored photos of the shirts in the study, a sample of three of them were recreated graphically for better print resolution (Figure 1).

We conducted individual semi-structured interviews with faculty and staff. ${ }^{19} \mathrm{We}$ also held semi-structured focus groups with the orientation committee members, and a mixture of individual interviews and focus groups with $2^{\text {nd }}-4^{\text {th }}$ year students. ${ }^{20} \mathrm{We}$ gave students the option to meet individually or in groups because we wanted to take student schedules into consideration. We began with face to face interviews in 2019, but during COVID-19 restrictions in 2020 we held seven interviews virtually with third year students. We were able to follow the same protocols, except for gaining oral rather than written participant consent.

For first year students, we used private documents as part of our design. ${ }^{18}$ Because these students had recently received their orientation t-shirts we wanted to explore the thoughts of as many as possible. Students wrote responses to prompts at the start of the school year focusing on the 2019 t-shirt they received, then responded to the remaining 20142018 shirts at end of school year. All students completed the writing to promote confidentiality but we only used writing from students who gave consent, with office staff collecting this information so we would not know who declined.

Sample interview and writing prompts are provided in Table 1. We conducted member checking to strengthen trustworthiness of the results. We returned transcripts to participants and gave them the opportunity to amend any comments. We conducted first cycle coding to establish categories by using a combination of structural and in vivo coding. ${ }^{21}$ For second cycle coding we used pattern coding to develop themes. ${ }^{21}$ The two authors independently coded data then met to discuss findings.

All participants chose pseudonyms for anonymity and the study was approved by Memorial University's Interdisciplinary Committee on Ethics in Human Research.

\section{RESULTS}


Data consist of interview and focus group data from faculty and staff, $2^{\text {nd }}-4^{\text {th }}$ year students, and orientation committee members; as well as writing from first year students. Details of the data and participants are provided in Table 2. Of note, all students had some part-time work or experiential education placements by the end of first year and 13/18 faculty and staff were pharmacists. Upon member checking, 12 participants replied verifying what they originally said, and one provided minor clarifications.

We developed five themes from our analysis. First, participants liked getting orientation t-shirts. Second, they thought medication and dispensary roles were predominant on the t-shirts. Third, not many thought the care provider relationship should have been emphasized. Fourth, there were mixed thoughts about the business logos; and fifth, students and faculty had many similar thoughts. These themes are detailed with quotes from the writing and interviews to contextualize and illustrate what participants thought and how they understood the graphics and images on the t-shirts. Participant chosen pseudonyms are used.

\section{Theme 1: Students and faculty liked the t-shirts as a welcoming gesture to the school and the profession.}

Students, faculty, and staff thought the main reason students are given orientation t-shirts is to welcome them to the school and the profession. Hippopotamus, a fourth year student, still remembered what it felt like, describing how "it's really scary on your first day of pharmacy school so it was something that kind of helped ease things and made it fun." Faculty member Amoxicillin similarly said it is a "mechanism of making them feel welcome and introducing them into the profession of pharmacy." This was usually the first type of comment made by all participants; that the t-shirts were meant to make students feel part of a group and connect with others. Hence, the practice of giving of shirts to first year students was considered positive.

\section{Theme 2: Students and faculty thought medication and dispensary roles were predominant on the t-shirts.}

Five of the six t-shirts had images of pills, a dispensary, or some form of drug expertise in a pop culture representation. Participants sometimes described this as the traditional role of pharmacists as opposed to the current role. Sabrina, a faculty member, noted one t-shirt was the "more traditional role of the pharmacist in the white lab coat outside of the pill bottles." Similarly, second year student Popcorn remarked, "it's just showing them behind the counter, probably just giving out medications," which was reiterated by Christmas Tree as, "back to when all they do is just stand behind the counter." Third year student Flower remarked about one shirt with a superhero image, "it's a pill... seems like the pills are maybe the superheroes rather than the pharmacists that give out the pills." Almost identically, Mary, a faculty member, expressed "it's a pill that's a superhero, so who has the superpowers, is it the pharmacist or is it the magic pill?"

As an extension of the emphasis on the dispensing role, some faculty did not think the shirts portrayed holistically what a pharmacist does. In fact, Bianca mentioned that "you know, around pharmacists only counting pills and filling prescriptions, obviously that's a perception that we want as pharmacists to dispel as much as possible." Capelin added, "there is no picture there of a pharmacist counselling. There is no picture there of a pharmacist doing an assessment on a patient, sitting down with a patient... There is no pharmacist there with a stethoscope hanging around his neck." Students also spoke to this, with McDouble, a first year student, writing, "pharmacists, obviously do most of their work concerning prescriptions, which is portrayed by the Rx." And Rad, a third year student, said regarding the pharmacists as a superhero, "as to how they do it, I guess, no, where the guys dressed up, he's a pill with a cape on. I guess that directly correlates to the drugs being what the pharmacists use, that's their superpower." Together, participants recognized the abundance of images about medications, prescriptions, and dispensing, yet did not think they were fully accurate relative to their thoughts about the profession. Further inquiry into how pharmacists' roles should be portrayed led to identification of the next theme.

\section{Theme 3: Fewer than half the students and faculty said the pharmacist patient relationship as in care provider should have been emphasized.}

Many students mentioned the pharmacist's role as helping people, especially in reference to the superhero themed shirts. For example, Capybara claimed that one shirt, "gives off the notion that we dispense medication and in doing so are aimed at helping people get better with whatever illness they have." Similarly, Duck mentioned, "so if I'm thinking that a pharmacist is like Superman because of that shirt, then they are very much talking to people, socializing with them to better their health." And Frank added, "I think we kind of build up the role as like something you know, we serve the public very thankless sometimes, so maybe the superhero comparison is really nice." Blueberry summed it up, stating that "pharmacists are dedicated to their jobs and live each day with the goal of helping patients with their medications."

Yet when asked what they would like to see on the t-shirts, only 15/37 students and faculty directly recommended that a pharmacist-patient interaction should be on the t-shirt. Three more mentioned expanded scope but did not elaborate and ten mentioned medications. Some faculty were in support of a patient interaction, as Christine proposed, "I would 
want to see more, something that symbolizes a relationship with a patient maybe, you know, patient and pharmacists conversing, or sitting down and talking about things. I think that will be a better depiction of a healthcare provider role." Similarly, Snow suggested "a handshake, or an information bullet coming from a pharmacist to another person," would emphasize communication. Photo Freak also pointed out the emphasis on drugs and suggested an emphasis on caring instead, "there are all kinds of things that pharmacists can do to care for people. That doesn't mean they have to give them a drug." Some students like Firefly, preferred an image without pharmacotherapy, and some like Tiramisu wanted "pharmacists in different roles besides a dispensary, like hospital, or industry, academia."

However, fewer than half emphasized the care provider role as being beyond medication expertise, as exemplified by several third year students. Mortar expressed, "you can put a syringe to symbolize vaccinations or injections.

Obviously, you got to have a pill." Frank wanted the mortar and pestle, because "I really like that symbology, I like the idea of a pharmacist as medicine creators." And Rad added "maybe some kind of imagery around being the drug expert," reinforcing the pharmacy identity of drug expertise. Hanna, a faculty member, suggested showing the impact of a pharmacist, but this too, was related to medications, such as "how many medication errors pharmacists can avoid." Faculty member Dora concluded, "all of the main messages they should know about their drugs and it's their responsibility to give it to patients, to provide that information to patients, that's their life. They're experts on it, they learn about it. That's their job."

\section{Theme 4: There were mixed reactions to the business logo on the t-shirt.}

Some participants saw the business logo as recognition of sponsorship and highlighting an investment in students. For example, Jane described it as "maybe some first years might see it as, wow, not only is the school welcoming but [business] is welcoming me." Steven, a second year student reiterated that sentiment, "they help us a lot, not with just orientation... they're so generous of how much they give to us," but was indifferent about an effect on them stating, "it probably does put [business] in a lighter or a positive view in my mind. Whether or not that's good or bad, and that could be me being a victim of advertising." Some faculty members had similar thoughts about advertising, with Dillman saying, "regardless of whether it was just intending to provide the shirt, the advertising was there, the name recognition, the name brands being established." Others also mentioned advertising but were not averse to this if arranged properly. For example, Mr. Science noted, "it's not just with orientation t-shirts, there's a broader issue, right? We get there's always a lot of sponsorship. We rely on that." Adding that it should be done in goodwill. And Hanna echoed, "I think it's only a tiny piece of a very broad exposure that they get, to sponsorship of events and things, especially during orientation week" but also expressed concern "that maybe the relationship is more formal than it appears because their branding is on our stuff."

Stella, a faculty member, outlined the dichotomy, stating, "I get it from a financial perspective instead of having to charge students for them because you want them to all have one and not everyone might be able to afford to have a tshirt." But also added that "it makes it seem like we are employees of [business]." Thirteen additional participants also mentioned the connection to employment. Meredith Grey wrote, "Having [business] on the back shows a reputable place where pharmacists work and can inspire students." Photo Freak added to the dichotomy with, "because here they are, they've got money. They're interested in me as a student because they're giving me a t-shirt."

Many of these comments were supplemented with the thought that it might induce bias as the same business sponsored all six t-shirts. Jenny, a fourth year student said it seemed like "we are really related to [business]. That was my first thought for this t-shirt." And Ralph, a second year student also thought, "it's like the school has some sort of thing with [business] going on, like we're going to send all of our students to you to work." And Bianca added, "I'm somewhat a little uncomfortable with this, because it's setting the student up to think that that's appropriate, when it may not be inappropriate, but it's not making them necessarily think about the influence of bias as they continue on their pharmacy education journey."

However, while there were cautions, participants acknowledged that pharmacy is a business, and most had no difficulties with its presence. Frank, a third year student explained, "you kind of maybe need to set aside your personal beliefs and do what's right in a business sometimes" and Yogurt, a faculty member reiterated, "I mean they are sponsored so we have the obligation to put their name there."

\section{Theme 5: There were more similarities than differences in what students and faculty thought.}

As illustrated in the previous themes, there were many similarities between what students and faculty thought. Regardless of years as student or faculty/staff member, many thoughts and feelings were the same. Most agreed the focus on medications represented a narrow view of pharmacists and would like to see a broader role symbolized. Fred Smith remarked, "I would describe the profession in a positive manner that really hits on certain myths about pharmacists, 
without getting involved in community pharmacy, or hospital, or the chains, just saying, you know, this is what pharmacists do, or can do within a day."

One notable difference was how the idea of pharmacists being superheroes was received. The first to third year students generally expressed pride and idealism in the profession, such as Anki writing, "we are powerful individuals/professionals like the Rx superhero, the symbol represents our knowledge, adaptability, and communication." However, some of the senior students and faculty were ambivalent about the portrayal. Lisa Simpson explained the superhero concept, "the thought behind it was pharmacists are superheroes, they take care of their patients and that's kind of the message we're trying to get across, but it really doesn't tell you why or how" and Pharmacy, a fourth year student added, "maybe they think that we give off a confidence, arrogance, like we're kind of on a high horse."

Some had comments about whether there was careful thought about pharmacist roles put into the shirts. Sabrina explained, "I think the students are more so trying to come up with an innovative, cool idea that represents pharmacy or pharmacy students without really thinking about what it means and what it portrays." Several t-shirt designers concurred, for instance, Coffee explained, "so you try things in general that even if you have no pharmacy knowledge at all, you would be able to think the shirt was cool." However, many participants also did not initially question the symbolism. For example, Dora, a faculty member said, "I don't see a link between the actual t-shirt themes and the sponsor" and Sue suggested "it doesn't give any perspective on what pharmacists do, or can do, or are capable of." Third year student Firefly initially said about one of the shirts, "I love the shirt, I'd wear it." Only upon further probing did participants begin to discuss the limited portrayal of pharmacist roles and relationships with business, as described in other themes. And, while most expressed some reservations about these portrayals, Reginald, a faculty member was not perturbed, "for the most part, these are good. They clearly have come out of the minds of upper year classes and there's nothing that I find overly concerning. Some of them are fun. Some of them are informative, but I think they serve the purpose."

\section{DISCUSSION}

Pharmacists thinking they should do more than dispense medications is not a recent idea. On the heels of a patient-centered pharmaceutical care philosophy, it is agreed worldwide that pharmacists have shifted from a product focus to patient care, from drug distribution to patient outcomes, in both community and hospital. ${ }^{22-24}$ Yet in surveys of hospital and community practices, most pharmacists continue to select a drug focus as the first choice for what a pharmacist does regardless of years of practice. ${ }^{25,26}$ As pharmacists cope with different identities and changing scopes of practice, desired and actual roles may differ. As such, our participants' thoughts about the t-shirt images may be reflective of the broader professional identity issues in pharmacy practice. Rutter et al. interviewed pharmacists in the United Kingdom with several pharmacists insisting they wanted more interactions with patients; but factors such as time, staff, etc. meant that dispensing and managing consumed most of their time. ${ }^{27}$ This, too, may be similar in pharmacy education. Educational outcomes for pharmacy programs encourage more than the dispensing role, yet the results of our study indicate that our participants' portrayals of pharmacy still center on drug expertise and provision, instead of care provider. Too, when asked to recommend images for orientation t-shirts, fewer than half the participants suggested images of the pharmacist as care provider. These results complement those of Burrows et al. who found that some students understood pharmacy practice from a narrow perspective that focused on the medications rather than patients, and may be reflective of a broader observation that the core functions of pharmacy in many countries continue to be dispensing and retail. ${ }^{11,28}$

Relatedly, the connection between pharmacy and business has led to a few contentious identities or roles of pharmacists. ${ }^{8,9}$ Codes of ethics for pharmacists in most professional regulatory bodies require that pharmacists avoid conflicts of interest that could affect their professional judgment and patient care. However, there is substantial literature focused on program interventions to foster business knowledge in pharmacy students. While some suggest industry influences could be problematic by biasing pharmacist decision-making and career choice, others emphasize improving student business acumen and describe sponsorship positively. ${ }^{29-32}$ Our results also indicated mixed thoughts and feelings about the possible negative influences of the retail business logo. With sponsorship came an expectation of acknowledgment on the t-shirt, but a few participants suggested a smaller image would be less obvious. It was interesting that although some recognized the possible biases that might be attached to the logo, it was generally considered acceptable.

To our knowledge, the underlying meanings associated with student selected graphics or symbols about pharmacy and pharmacist roles have not been comprehensively studied. Our study explored the thoughts of a broad range of participants by including students in all years of study as well as faculty. We recognize that our participants' understandings may reflect the social environment of pharmacy school and practice in one particular jurisdiction. However, considering that this practice of giving orientation shirts is present in most Canadian pharmacy schools, we think our study may serve as a starting point for pharmacy educators to think carefully about the symbols and images used in pharmacy education. One limitation for our study was that we did not examine the discursive practices that might have 
influenced our participants' thoughts about pharmacists' roles; we were seeking to gain understanding of their emerging identity associations with being or becoming a pharmacist. Further research needs to be conducted to uncover why students and faculty think the way they do. A second limitation, or rather a delimitation, is that we only examined orientation t-shirts, we did not study any other artifacts that might contribute to student and faculty perceptions about pharmacist identity. Last, we did not collect data on how the images on the t-shirts might influence the thoughts of students who receive them about pharmacists' identities.

\section{CONCLUSION}

Understandings and perceptions of images on orientation t-shirts in our study helped to illuminate aspects of the hidden curriculum and discursive practices that form beliefs and identities. Mr. Science emphasized the importance of this study with this concluding comment, "I do think it's important that you're doing this because this [images] does have an impact on students." Our study used the images to question how students whose explicit curriculum is based in patientcentred care come to create particular representations of pharmacists' roles. We showed that despite national educational outcomes advocating the care provider role for pharmacists, these representations of pharmacy still focus on drug expertise and drug provision, and apart from our probing, these pharmacy students and faculty did not appear to question this symbolism. The idea that students and faculty were accepting of these images without questioning is a concern, given the expanding scope of pharmacy practice that emphasizes the role of care provider. We need to be thinking about the messages we portray, regardless of whether they are implicit or explicit. We conclude with fourth year Pizza's summation, an enlightening reflection on the effect of discursive practices of pharmacy education, "mostly we focus on medications, so it's good to highlight we're dealing with medication."

\section{REFERENCES}

1. Association of Faculties of Pharmacy of Canada. Educational Outcomes for First Professional Degree Programs in Pharmacy in Canada.; 2017. https://www.afpc.info/node/39

2. Medina MS, Plaza CM, Stowe CD, et al. Center for the advancement of pharmacy education 2013 educational outcomes. Am J Pharm Educ. 2013;77(8):162. doi:10.5688/ajpe778162

3. Mahood SC. Medical education: Beware the hidden curriculum. Can Fam Physician. 2011;57(9):983-985.

4. Martimianakis MA (Tina), Michalec B, Lam J, Cartmill C, Taylor JS, Hafferty FW. Humanism, the hidden curriculum, and educational reform: A scoping review and thematic analysis. Acad Med. 2015;90(11):S5-S13. doi:10.1097/ACM.0000000000000894

5. van Huyssteen M, Bheekie A. The hidden curriculum of work-based learning for pharmacy students in public sector pharmacies in South Africa. Pharm Educ. 2017;17(1):190-198.

6. $\quad$ Foucault M. The Archaeology of Knowledge and the Discourse on Language. Vintage Books; 1972.

7. Bacchi C, Bonham J. Reclaiming discursive practices as an analytic focus: Political implications. Foucault Stud. 2014;17(17):173-192.

8. Kellar J, Paradis E, van der Vleuten CPM, Oude Egbrink MGA, Austin Z. A historical discourse analysis of pharmacist identity in pharmacy education. Am J Pharm Educ. 2020;84(9):1251-1258. doi:10.5688/ajpe7864

9. Elvey R, Hassell K, Hall J. Who do you think you are? Pharmacists' perceptions of their professional identity. Int $J$ Pharm Pract. 2013;21(5):322-332. doi:10.1111/ijpp.12019

10. Atkinson J, de Paepe K, Sánchez Pozo A, et al. What is a Pharmacist: Opinions of Pharmacy Department Academics and Community Pharmacists on Competences Required for Pharmacy Practice. Pharmacy. 2016;4(1):12. doi:10.3390/pharmacy4010012

11. Burrows J, Dall'Alba G, Caze A La. Becoming pharmacists: Students' understanding of pharmacy practice at graduation from an Australian University. Curr Pharm Teach Learn. 2016;8(6):729-741. doi:10.1016/j.cpt1.2016.08.007

12. Gregory P, Austin Z. Pharmacists' lack of profession-hood: Professional identity formation and its implications for practice. Can Pharm J. 2019;152(4):251-256. doi:10.1177/1715163519846534

13. Dawodu P, Rutter P. How Do Pharmacists Construct, Facilitate and Consolidate Their Professional Identity? Pharmacy. 2016;4(3):23. doi:10.3390/pharmacy4030023

14. Stieb EW. Symbol of pharmacy. J Am Pharm Assoc. 1962;2(4):206-209. doi:10.1016/s0003-0465(15)30276-7

15. Sonnedecker G. Origin of the AIHP Emblem. Pharm Hist. 1995;37(1):28-31.

16. Bailey E. Symbols: Historic and current uses. Int J Pharm Compd. 2008;12(6):505-507.

17. Noble C, O'Brien M, Coombes I, Shaw PN, Nissen L, Clavarino A. Becoming a pharmacist: Students' perceptions of their curricular experience and professional identity formation. Curr Pharm Teach Learn. 2014;6(3):327-339. doi:10.1016/j.cpt1.2014.02.010 
18. Creswell J. 30 Essential Skills for the Qualitative Researcher. SAGE Publications; 2016.

19. Brinkmann S, Kvale S. Interviews: Learning the Craft of Qualitative Research Interviewing. 3rd ed. SAGE Publications; 2015.

20. Krueger RA, Casey MA. Focus Groups: A Practical Guide for Applied Research. 4th ed. SAGE Publications; 2009.

21. Saldaña J. The Coding Manual for Qualitative Researchers. 3rd ed. SAGE Publications; 2015.

22. Kritikos V, Watt HMG, Krass I, Sainsbury EJ, Bosnic-Anticevich SZ. Pharmacy students' perceptions of their profession relative to other health care professions. Int J Pharm Pract. 2003;11(2):121-129. doi:10.1211/0022357021242

23. Schindel TJ, Yuksel N, Breault R, Daniels J, Varnhagen S, Hughes CA. Perceptions of pharmacists' roles in the era of expanding scopes of practice. Res Soc Adm Pharm. 2017;13(1):148-161. doi:10.1016/j.sapharm.2016.02.007

24. Anderson S. The state of the world's pharmacy: A portrait of the pharmacy profession. J Interprof Care. 2002;16(4):391-404. doi:10.1080/1356182021000008337

25. Al Hamarneh YN, Rosenthal M, McElnay JC, Tsuyuki RT. Pharmacists' perceptions of their professional role: Insights into hospital pharmacy culture. Can J Hosp Pharm. 2011;64(1):31-35. doi:10.4212/cjhp.v64i1.984

26. Rosenthal MM, Breault R, Austin Z, Tsuyuki RT. Pharmacists' self-perception of their professional role: Insights into community pharmacy culture. J Am Pharm Assoc. 2011;51(3):363-367. doi:10.1331/JAPhA.2011.10034

27. Rutter PR, Hunt AJ, Jones IF. Exploring the gap: Community pharmacists' perceptions of their current role compared with their aspirations. Int J Pharm Pract. 2000;8(3):204-208. doi:10.1111/j.2042-7174.2000.tb01006.x

28. Mossialos E, Courtin E, Naci H, et al. From "retailers" to health care providers: Transforming the role of community pharmacists in chronic disease management. Health Policy (New York). 2015;119(5):628-639. doi:10.1016/j.healthpol.2015.02.007

29. Slavcev RA, Waite NM, Jennings B. Shaping pharmacy students' business and management aptitude and attitude. Curr Pharm Teach Learn. 2016;8(5):672-680. doi:10.1016/j.cpt1.2016.06.003

30. Augustine J, Slack M, Cooley J, Bhattacharjee S, Holmes E, Warholak TL. Identification of key business and management skills needed for pharmacy graduates. Am J Pharm Educ. 2018;82(8):983-991. doi:10.5688/ajpe6364

31. Piascik P, Bernard D, Madhavan S, Sorensen TD, Stoner SC, Tenhoeve T. Gifts and corporate influence in doctor of pharmacy education. Am J Pharm Educ. 2007;71(4). doi:10.5688/aj710468

32. Robertson J, Walkom E, Moynihan R, Bero L, Henry D. Pharmaceutical industry funding of educational events for pharmacists in Australia: An analysis of data from the first 6 months of a mandatory disclosure programme. Int $J$ Pharm Pract. 2010;18(2):88-92. doi:10.1211/ijpp/18.02.0003 
Sample Prompts

Writing activity for first year students

Interviews and focus groups with students, faculty, and staff
Why do you think first year pharmacy students are given t-shirts as part of orientation?

What message(s) do you take away about the role of pharmacists from the symbols and/or graphics on these t-shirts?

Why do you think first year pharmacy students are given t-shirts as part of orientation? What do the images on each shirt tell you a pharmacist does?

As upper year students with some experience (or as faculty/staff), what do you think about the messages you've identified?

Are these messages what you want to see given to first year students?

If you were to design a t-shirt for first year students, what imagery would you put on it? Do you see any connections between pharmacy and business in these t-shirts?

Table 2. Data and Participant Characteristics

\begin{tabular}{lccc}
\hline & $\begin{array}{c}\text { Number of participants } \\
\text { (N=86) }\end{array}$ & $\begin{array}{c}\text { Minutes of interview or } \\
\text { focus groups } \\
\text { (Total=1144 minutes) }\end{array}$ & $\begin{array}{c}\text { Pages of writing or } \\
\text { transcription } \\
\text { (Total=437 pages) }\end{array}$ \\
\hline First year students & $39\left(34^{\mathrm{a}}\right)$ & N/A & 107 \\
Second year students & 8 & 99 & 48 \\
Third year students & 11 & 217 & 58 \\
Fourth year students & 6 & 67 & 25 \\
Orientation committee & 4 & 53 & 24 \\
Faculty and staff & 18 & 708 & 175 \\
\hline
\end{tabular}

${ }^{\text {a }}$ Number of participants for end of year writing activity was less as some students were absent

Figure 1. Sample of Three Orientation T-Shirts Used for Discussion

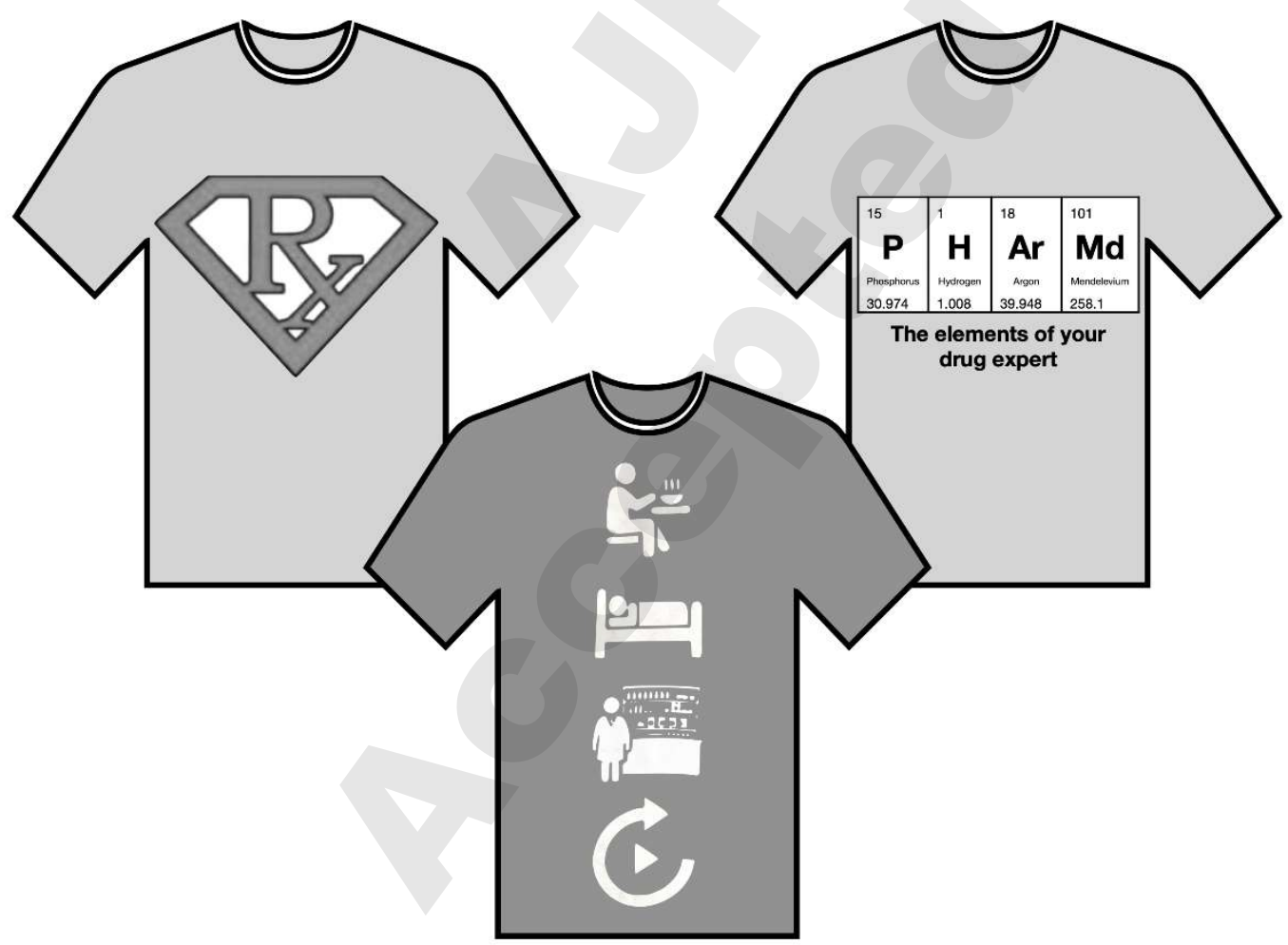

\section{Legend}

Orientation t-shirts recreated graphically for print. The 2016 shirt was a play on the Superman logo and the 2017 shirt had periodic table elements placed together to spell PharmD. Sponsor logos were prominently displayed on the back of the shirts but are not shown here to maintain confidentiality. 